\title{
DAB Based DC-DC High Frequency Link PET for Interconnecting MVDC-LVDC Grids
}

\author{
D.Srinivasa $\operatorname{Rao}^{1} \mid$ Dr. Anupama A. Deshpande ${ }^{2}$ \\ ${ }^{1}$ Department of Electrical Engineering, Shri JJTUniversity, Jhunjhunu, Rajasthan, INDIA. \\ ${ }^{2}$ Department of Electrical Engineering, Shri JJTUniversity, Jhunjhunu, Rajasthan, INDIA.
}

To Cite this Article

D.Srinivasa Rao \& Dr. Anupama A. Deshpande. DAB Based DC-DC High Frequency Link PET for Interconnecting MVDC-LVDC Grids. International Journal for Modern Trends in Science and Technology 7, 165-171 (2021).

\section{Article Info}

Received on 25-April-2021, Revised on 15-May-2021, Accepted on 23-May-2021, Published on 26-May-2021.

\section{ABSTRACT}

This paper proposes dual active bridge (DAB) based high frequency power electronic transformer (PET) for interconnecting medium voltage $d c(M V D C)$ and low voltage dc (LVDC) grids for dc power distribution. The above proposed concept works on dual active phase shift principle and square wave HF modulation technique for bidirectional power transfer. Compared to the traditional dc transformer scheme, The proposed power electronic transformer (PET) can disconnect from LVDC distribution grid effectively as a dc breaker when a short circuit fault occurs in the distribution grid. The isolated DC-DC PET topology with a wide range of voltage conversion ratio is useful for High Voltage DC tapping. The DAB based on switched capacitor is connected to the medium voltage DC side and acts as an inverter. The proposed topology has the ability to transfer higher power, and lower circulating power, lower high frequency link voltage, and RMS current and peak values with the same transmission power in the MVDC side. This paper analyzes the topology, voltage and power characterization, control strategy in detail. Increase in the intermediate AC frequency will reduce the size of the transformer and other passive elements significantly in the circuit. The theoretical analysis is supported by MATLAB simulation.

KEYWORDS: $D A B, P E T, L V D C, M V D C$, high frequency link.

\section{INTRODUCTION}

Medium voltage dc (MVDC) distribution grid is an intermediate link in building modern dc grid especially to connect high-voltage dc (HVDC) transmission grid and low voltage dc (LVDC) distribution micro grid [1]-[2].

In dc power distribution, recent research mainly focuses on the low-voltage dc (LVDC) microgrid, and the flexible HVDC transmission is also developing rapidly, high voltage or medium voltage (HV/MV) distribution became prominent. In medium-voltage dc (MVDC) distribution, to achieve the voltage conversion and electrical isolation between MVDC distribution bus and LVDC microgrid bus, a dc transformer is required [3]-[4]. In dc distribution, it is difficult to realize power conversion through a simple magnetic transformer; it really needs power electronics technology. In LV applications, dc-dc converters are widely used. Many literatures discussed isolated bidirectional dc-dc converters, which are required for achieving electrical isolation. These converters can be used to interface dc loads, energy storage systems, and dc distributed generations in the LV side, particular in considering integration of renewable energy [5]. The main objectives are usually buck/boost circuits or isolated dc-dc, and the output voltage of these converters is controlled by duty ratio of switches. These types of dc converters should meet the following requirements such as bidirectional power 
flow, electrical isolation, high-power application, etc.

The DAB topology is the most suitable converter cell, which can be used as a base cell for PET. On this basis, multiple DABs are used in PET [6]. Even though previous multiple PET topology based on DAB can achieve electrical isolation, voltage conversion and power transfer between MVDC distribution grid and LVDC microgrid buses. Due to the existence of the concentrated capacitor, The DAB module cannot disconnect from MVDC distribution when a short fault occurs in the distribution bus, the dc capacitor will be discharged rapidly and there will be a large over current [7]. When the fault is eliminated, the dc capacitor needs to be recharged, and the fault recovery is very slow. The circulating power of DAB increases sharply when the terminal voltages mismatched with the transformer ratio, the efficiency of PET decreases rapidly when the distribution voltage fluctuation occurs [8]-[9].

Considering such situations mentioned earlier, this paper proposes a PET based on switched capacitor to address the mentioned issues. The number of sub modules in an SC based PET can be adjusted according to the voltage and power levels, and it is especially suitable for KV and MW level applications with large no of sub modules to improved fault tolerance, reliability and efficiency. The rest of the paper is organized as follows. Section II discusses the proposed topology based on switched capacitor based DAB modules. Section III emphasizes the detailed characterization of the mentioned PET for MVDC distribution application. Section IV discusses the control characterization and design of the PET. Section $V$ gives MATLAB simulation results to support theoretical analysis. Section VI gives conclusion and the future scope of the paper.

\section{PROPOSED PET BASED ON SWITCHED CAPACITOR}

The topology of a proposed PET is shown in Fig. 1. The PET is mainly composed of a series switched capacitor interface (SSCI) and high-frequency link interface (HFLI). It is composed of $N$ Half bridge (HB) switched capacitor (SC) sub modules, then $N$ discrete dc terminals $\left(V_{C 1}, V_{C 2}, \ldots, V_{C N}\right)$ are created as shown in Fig. 1; all the SCs are connected in series at the ac side to interface the MVDC distribution grid. The high-frequency link interface comprised of N DAB sub modules. One terminal of $\mathrm{DAB}$ connected to the discrete dc terminal of SC and the other connected in parallel to interface the LVDC microgrid port.

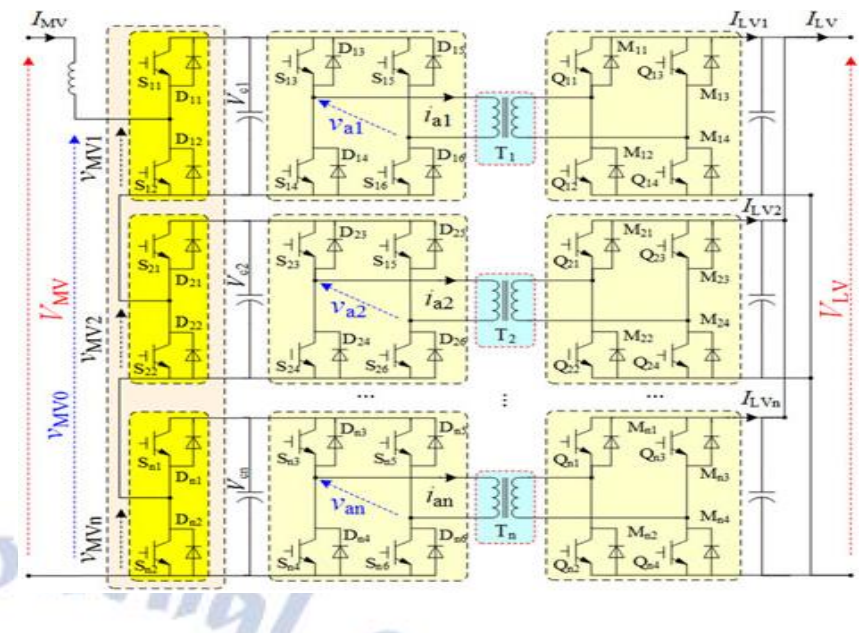

Fig. 1. Topology of proposed PET.

In practice, the transformers in traditional PET are connected in series and in proposed PET topology are distributed to each $\mathrm{DAB}$, that just reduces the operating voltages of primary and secondary sides of transformer are not higher [10]-[11]. In the proposed PET topology, each SM of the MMC leg can be operated as a separate buck/boost converter; then one SM and one DAB can be seen as a two-stage dc-dc converter. The balance control of the MMC leg can also be incorporated in the corresponding DAB cell, so the control of PET has more modularity. Every cell of PET can operate independently and also in series to connect the MVDC bus, which decreases the difficulty of installation and commissioning. The proposed PET employs series switched capacitor interface as an interface to the MVDC distribution grid. Series switched capacitor interface can adjust the voltage of the dc discrete terminals to ensure the voltage match between the two sides of the HFL transformer [12]-[13]. Then the circulating current can be decreased and the current impact and efficiency performances can be improved. The PET can operate well even if some sub modules fail, and then the reliability of the PET can be improved. Also the dc discrete voltages can stay when the MVDC distribution grid fails as the SCs could not discharge, and then the PET can put into operation quickly when the fault is eliminated [14]-[15]. All modules are connected in parallel on the LV side. When a sub module fails, we just need to close the driving pulses of all the switches of those faulty modules only. The LV side of the PET operates normally. 


\section{CHARACTERIZATION OF PET FOR MVDC DISTRIBUTION}

\section{A. Dual-Active Phase-Shift Principle}

Dual-active phase-shift principle is employed to transfer power, as shown in Fig.2. For PET, the whole DAB module is equivalent to two HF waves $v_{a}$ and $v_{b}$ connected with an inductor. For PET, each cell is equivalent to two HF waves $v_{a i}$ and $v_{b i}$ connected with an inductor. Then, the magnitude and direction of power flow can be adjusted by controlling the magnitude and direction of phase-shift angle between two HF waves. According to the analysis in previous literatures, the square modulation has higher power transfer ability, better current performance, and simpler control method. So, the PET can just employ square modulation only.

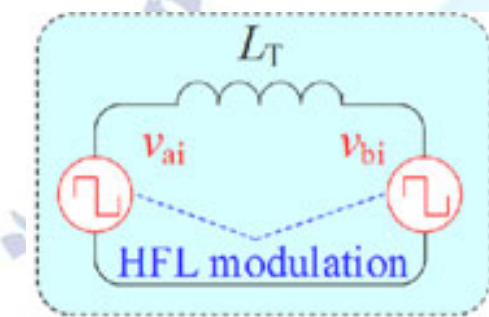

Fig.2. Dual-active phase-shift principle for PET

\section{B. Switching Characterization}

In series switched capacitor modules, the two switches Qi1 and Qi2 are switched alternately and the switching states of the switches in the same position of all the SC sub modules are the same, as shown in Fig. 3.

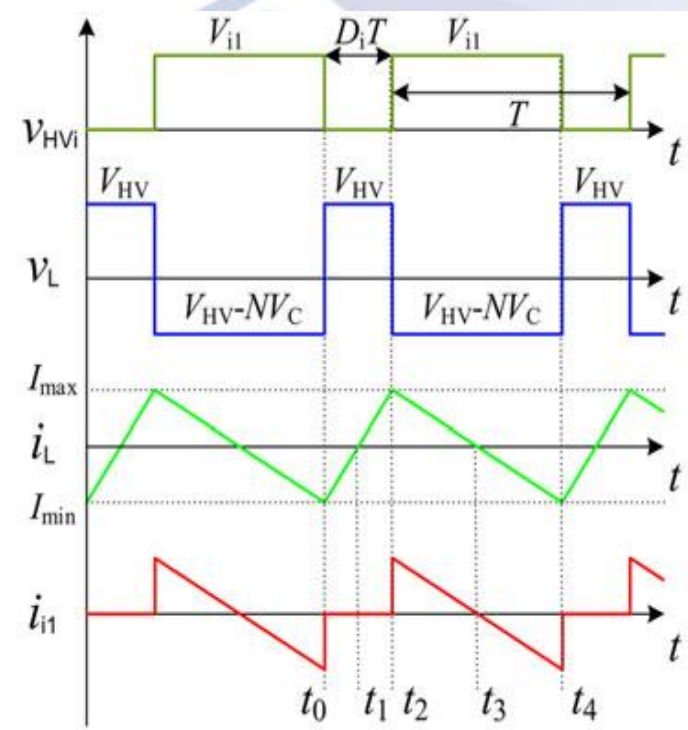

Fig.3. Waveforms of PET for SC modules
From Fig. 3 the operation of series SC modules can be divided into four modes.

Mode.1. During interval $t_{0}-t_{1}: S_{i 1}$ is turned off and $S_{i 2}$ is turned on, and the inductor current $i_{L}$ is negative. Then $i_{L}$ flows through $D_{i 2}$, and the voltage across $L$ is $V_{H V}$. The current $i_{L}$ decreases in the negative direction until $i_{L}=0$ at $t_{1}$.

Mode.2. During interval $t_{1}-t_{2}: S_{i 1}$ is still turned off and $S_{i 2}$ is still turned on, but the inductor current $i_{L}$ is changed from negative to positive. Then $i_{L}$ flow shift from $D_{i 2}$ to $S_{i 2}$, and the voltage across $L$ is still $V_{H V}$; the current $i_{L}$ increases in the positive direction until $i_{L}$ achieves the maximum value $I_{\max }$ at $t_{2}$.

Mode.3. During interval $t_{2}-t_{3}$, at $t_{2}, S_{i 1}$ is still turned on and $S_{i 2}$ is still turned off, and the inductor current $i_{L}$ is positive. Then $i_{L}$ flows through $D_{i 1}$, and the voltage across $L$ is $V_{H V}-\sum V_{i 1}$; the current $i_{L}$ decreases in the positive direction until $i_{L}=0$ at $t_{3}$.

Mode.4. During interval $t_{3}-t_{4} S_{i 1}$ is still turned on, and $S_{i 2}$ is still turned off, but the inductor current $i_{L}$ is changed from positive to negative. Then $i_{L}$ flow shifts from $D_{i 1}$ to $S_{i 1}$, and the voltage across $L$ is still $V_{H V}-\sum V_{i 1}$; the current $i_{L}$ increases in the negative direction till $i_{L}$ achieves the minimum value $I_{\min }$ at $t_{4}$.

In HFL DAB , the switching states of $S_{i 3}, S_{i 5}, Q_{i 1}$ and $Q_{i 3}$ are complimentary with $S_{i 4}, S_{i 6}, Q_{i 2}$ and $Q_{i 4}$, respectively; the switching states of $S_{i 3}, S_{i 5}, S_{i 4}$, $S_{i 6}$ are same with $Q_{i 1}, Q_{i 3}, Q_{i 2}$ and $Q_{i 4}$ respectively; the switching states of the switches in the same position of all the DAB sub modules are the same. In the steady state, HFL DAB can be divided into six modes as shown in Fig.4.

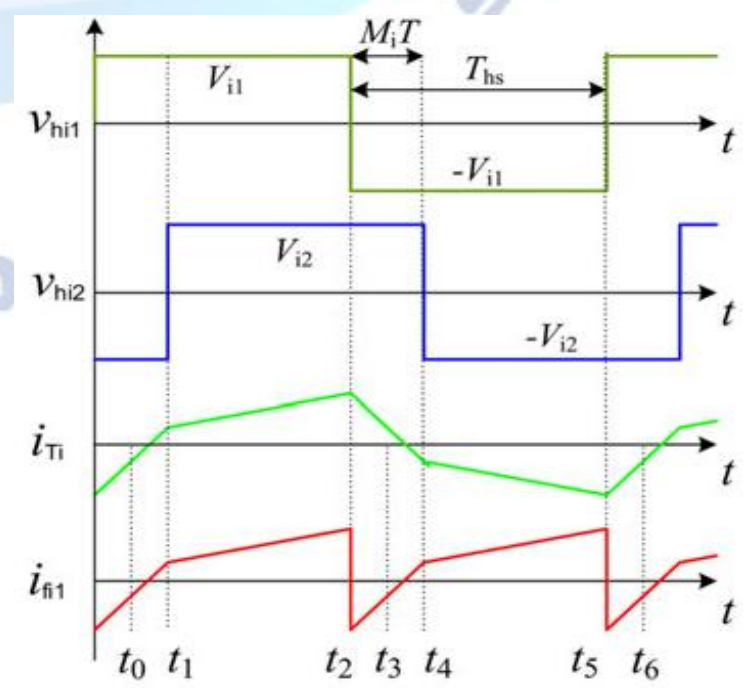

Fig.4. Waveforms of PET for DAB modules 


\section{Voltage and Power Characterization of PET}

According to the analysis mentioned, the average voltage of $L$ during one period can be derived as follows:

$V_{L}=\frac{1}{T} \int_{t_{0}}^{t_{4}} v_{L} d t=V_{H V}-\sum_{i=1}^{i=n}\left(1-D_{i}\right) V_{i 1}$

Where $D_{i}=\left(t_{2}-t_{0}\right) / T$ is defined as the duty ratio and $T$ is the switching period of series SC modules. When all the duty ratios are the same, the average voltage of the inductor $L$ over one period of time T should be zero during the steady state. From (1), we have

$V_{C a v}=\frac{\sum_{i=1}^{i=n} V_{i 1}}{N}=\frac{V_{H V}}{N(1-D)}$

Where $V_{C \text { av }}$ the average value of all the dc discrete voltages of series switched capacitor modules, and $D=D_{1}=D_{2}=\ldots=D_{N}$.

From (2), the capacitor voltage of SC can be adjusted by adjusting the duty ratio $D$. Due to $0 \leq D$ $<1$, thus $V_{C \text { av }} \geq V_{H V} / N$. In addition, the average value of $i_{L}$ and $i_{i 1}$ over one switching period also can be derived as

$I_{L}=\frac{1}{T} \int_{t_{0}}^{t_{4}} i_{L} d t=\frac{I_{\max }+I_{\min }}{2}$

$I_{i 1}=\frac{1}{T} \int_{t_{2}}^{t_{4}} i_{L} d t=\frac{(1-D)\left(I_{\max }+I_{\min }\right)}{2}$

Omitting the power loss, the average power of series switched capacitor modules is

$\mathrm{P}=V_{H V} I_{H V}=V_{H V} I_{L}=\sum_{i=1}^{i=n} V_{i 1} I_{i 1}=\frac{\left(I_{\max }+I_{\min }\right)}{2} V_{H V}$

From (4), $P=0$ while $I_{\max }+I_{\min }=0$, there is no power flow between series switched capacitor modules and HFL DAB; If $P>0, I_{\max }+I_{\min }>0$, the power flow between series switched capacitor modules to HFL DAB, If $P<0, I_{\max }+I_{\min }<0$, the power flows from HFL DAB to SC module.

From Fig. 4, each DAB can be equivalent to two ac voltages $v_{h i 1}$ and $v_{h i 2}$ connected to both sides of an inductor; the magnitude and direction of the power can be adjusted by adjusting the magnitude and direction of phase shift (PS) between $v_{h i 1}$ and $v_{h i 2}$. The average power of DAB can be derived as

$$
P_{i}=\frac{n_{T} V_{i 1} V_{i 2}}{2 f_{S} L_{T}} M_{i}\left(1-\left|M_{i}\right|\right)
$$

Where $M_{i}=\left(t_{4}-t_{2}\right) / T_{h s}$ is defined as the PS ratio, $0 \leq M_{i} \leq 1 ; f_{s}=1 /\left(2 T_{h s}\right)$ is the switching frequency; and $n_{T}$ is the turns ratio of the transformer.

For HFL DABs, the dc currents at the series side of all the DABs are the same and the dc voltages at the parallel side are the same.

When all the PS ratios are the same, we have

$\mathrm{P}=\sum_{i=1}^{N} P_{i}=\frac{n_{T} N V_{C} V_{L V}}{2 f_{S} L_{T}} M(1-|M|)$

Where $\mathrm{M}=M_{1}=M_{2}=\cdots M_{N}$

From (2) and (6), we have

$\mathrm{P}=\frac{n_{T} V_{H V} V_{L V}}{2 f_{S} L_{T}} \frac{M(1-|M|)}{(1-D)}$

$V_{L V}=\frac{n_{T} V_{H V} R}{2 f_{S} L_{T}} \frac{M(1-|M|)}{(1-D)}$

According to the above given analysis, the voltage and power of PET can be controlled by controlling the duty ratio $D$ and the PS ratio $M$. From (7) when the PS ratio $M>0$, the transmission power is positive; when the PS ratio $M<0$, the transmission power is negative. When $|M|<0.5$, the transmission power increases with the increase in $|M|$ and $D$. It also evident that the PET has greater transmission power capacity than traditional DC transformer because of employing series switched capacitor modules.

\section{Circulating Current Characterization of PET}

In the HF link, because of the power transferred by $\mathrm{HF}$ square waves in every $\mathrm{DAB}$, the circulating current will increase rapidly when the terminal voltages do not match to the turns ratio of the transformer.

The circulating current performance of $\mathrm{DAB}$ can be shown by the HF link fundamental power factor, as analyzed and then

$\lambda_{P E T}=\frac{n_{T} V_{L V} \sin (M \pi)}{\sqrt{\left.\frac{\left\{V_{H V}\right.}{[N(1-D)]}-n_{T} V_{L V} \cos (M \pi)\right\}^{2}+\left[n_{T} V_{L V} \sin (M \pi)\right]^{2}}}$

It can be seen that the PET can get larger power factor than the traditional dc transformer in all the operation ranges by selecting the appropriate duty ratio of series SC modules, and then the circulating current will be decreased and the efficiency can be improved. The decrease in the circulating current will lead to decrement in the RMS and peak currents, so both the conduction and switching losses will be reduced. 


\section{CONTROL CHARACTERIZATION AND DESIGN OF THE PET}

In PET, the MVDC voltage is provided by the dc distribution grid, and the LVDC voltage is controlled by the SC dc transformer. The importance of switched capacitor dc transformer is to control the voltage balance of discrete capacitors in series and current balance of each DAB in parallel on the LV side for both the forward and reverse power flows.

\section{A. Self-Balancing Capacity}

The average value of the dc discrete voltage is controlled with a constant value by series SC module and the LVDC voltage is controlled with a constant value by HFL DAB. From (2) to (7), $D, M, i_{L}$ , and Ii1 remain constant for a given transmission power.

From Fig. 1, for SCi and DABi, we have

$I_{i C 1}=I_{i 1}-I_{i f 1}$

Let the discrete voltage $V 11$ of $\mathrm{SC} 1$ increases and the discrete voltage $V 21$ of SC2 decreases due to the disturbance. Because of $V L V$ and $M$ are remains constant, the power $P_{1}$ of DAB1 will increase and the power $P_{2}$ of DAB2 will decrease from (5). Because the LVDC side voltages of DAB1 and DAB2 are the same, the parallel current $I_{1 f 2}$ will increases and $I_{2 f 2}$ decreases, and then $I_{1 f 1}$ will increases and $I_{2 f 1}$ decreases due to the fixed turns ratio of the HF transformer.

From (9), the capacitor current $I_{1 C 1}$ of SC1 will decrease and the capacitor current $I_{2 C 1}$ of SC2 will increase, because the capacitor current is zero in the steady state, $I_{1 C 1}$ current become negative and $I_{2 C 1}$ will become positive, that is, capacitor $C_{11}$ is discharged and capacitor $C_{21}$ is charged. Then, the discrete voltage $V 11$ of SC1 will decrease and the discrete voltage $V 21$ of SC2 will increase and the $\mathrm{PET}$ will be recovering the balance automatically.

\section{B. Stability of Voltage and Current Balancing Control}

Even though the PET has some self-balancing capacity, the balancing control should to be added to ensure the voltage balancing of discrete capacitors and the current balancing of each DAB in all the possible situations. In steady state, the average current through the discrete capacitor during one period of time is zero, from (9), we have
$I_{i C 1}=0, I_{i 1}=I_{i f 1}=(1-D) I_{H V}$

Omitting the power loss in the PET, the input and output powers of DABi are the same and then

$$
P_{i}=V_{i f 1} I_{i f 1}=V_{i f 2} I_{i f 2}
$$

Due to the currents of the PET in the series side are the same and the voltages in the parallel side are also same, we have

$V_{11}=V_{21}=V_{31}=\ldots=V_{N 1}$

$I_{1 f 2}=I_{2 f 2}=I_{3 f 2}=\ldots=I_{N f 2}$

$P_{1}=P_{2}=P_{3}=\ldots=P_{N}$

From (12), the voltage balance in the series side and the current balance in the parallel side can lead to the power balance of the PET even the two methods have different control stabilities. Due to the control in the series voltage balance, from (5) the powers developed by all the DABs are equal due to the equal voltage in the parallel side, so the currents in the parallel side are also equal.

From (9) and (11), the dynamic control in the series side is shown in Fig. 5(a), the control for the parallel current balance is employed. In the initial state, the DABi operates stably, thus, $V i 1=V \mathrm{C}$ av, $I_{i f 1}=I_{i 1}$, and $I_{i C 1}=0$; because of the parallel currents are controlled to equal values, so the power developed by of all the modules are also equal.
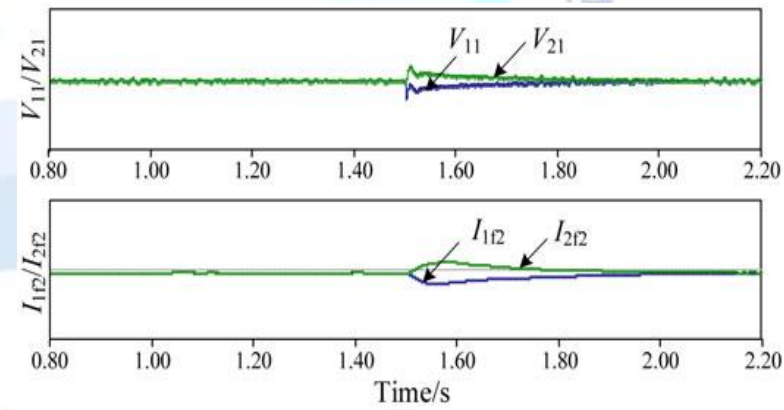

(a)

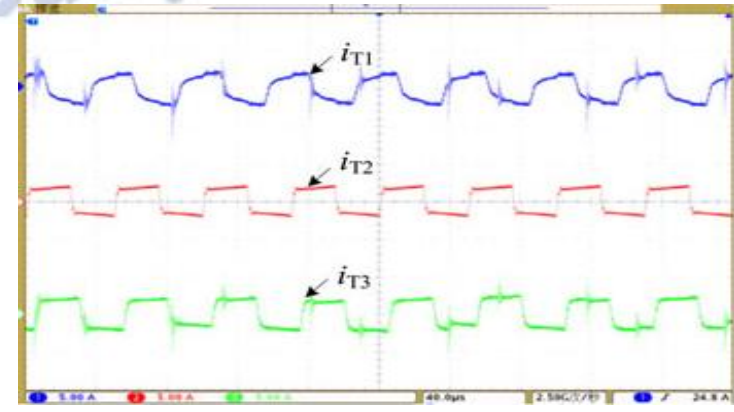

(b)

Fig.5. (a).Self balance control of voltage of PET (b). Balanced transformer currents 


\section{Control Design of PET}

Generally PET is a bidirectional system, to ensure the stable operation in the forward and reverse power flow, the control for the voltage balance in the series side is employed in the paper.

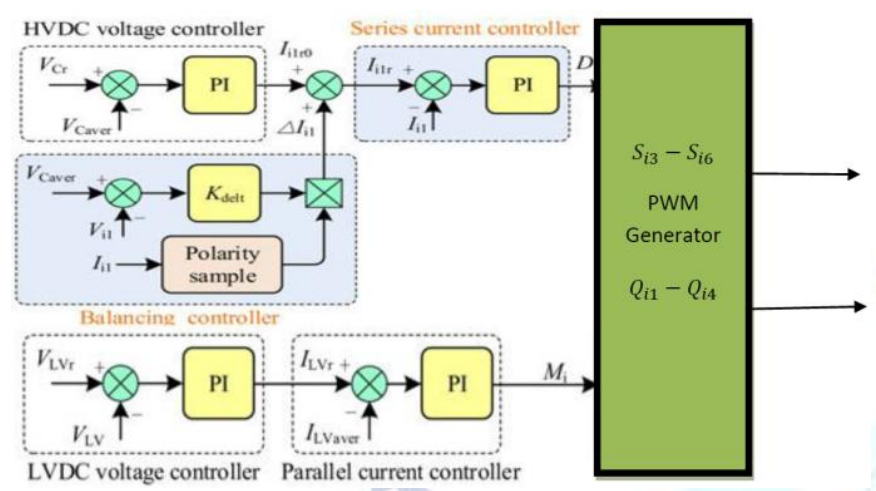

Fig.6. Balance control of PET

Fig.6 shows a control strategy for PET. From Fig.6, the dc step voltages are controlled by series SC modules and the LVDC voltage is controlled by HFL DAB. In series $\mathrm{SC}$ modules, the HVDC voltage controller takes the difference value between the sampled average value $V C$ av and the set reference value $\mathrm{VCr}$ to the PI controller, and the output of the PI controller is used as a reference value $I i 1 r 0$ of the series current controller. The balancing controller samples the dc step voltage $V_{1} 1$ and then compares $V C$ av and $V i 1$ to calculate the correcting value $\Delta I i 1$ of the unified reference current $I i 1 r 0$. The actual reference current Ii1 $r$ is the synthesis of $I i 1 r 0$ and $\Delta I i 1$. Then the series current controller calculates the duty ratio $D i$ of each SC according to Ii1 $r$ and the sampled series current $l i 1$.

In HFL DAB, the LVDC voltage controller takes the difference value between the sampled voltage $V L V$ and set reference value $V L V r$ to the PI controller and the output of the PI controller is used as the reference current $I L V \quad r$ of the parallel current controller. The parallel current controller samples all the currents in the parallel side and calculates their average value $I L V$ av. Then the PS ratio $M i$ is achieved by the adjustment of the PI controller.

$V \mathrm{C}$ av $=\frac{\sum_{i=1}^{N} V_{i 1}}{N}, \operatorname{LV}$ av $=\frac{\sum_{i=1}^{N} I_{i 2}}{N}$

\section{SIMULATION VERIFICATION}

To verify the theoretical analysis mentioned earlier, a 1-kW PET MATLAB prototype with three sub modules is built, where the MVDC and the LVDC rated voltages are $V H V=400 \mathrm{~V}$ and $V L V=200 \mathrm{~V}$ respectively, the dc inductor $L=5 \mathrm{mH}$, the dc capacitors $\mathrm{Ci} 1=\mathrm{Ci} 2=\mathrm{Ci} 3=3300 \mu \mathrm{F}$, the $\mathrm{HFL}$ inductor $L_{T}=100 \mu \mathrm{H}$, the transformer ratio $n=1: 1$, and the switching frequency $f_{s}=20 \mathrm{kHz}$.

Fig. 7 shows the voltage and current waveforms of PET in the steady state. It can be seen that the MVDC and the LVDC buses stay at $400 \mathrm{~V}$ and $200 \mathrm{~V}$ respectively, both the current voltage conversions of PET operates as estimated. All the voltages of discrete capacitors are equal, which indicates a well voltage balanced effect; all the HFL currents of each DAB are also equal, which indicates a well power balance effect.

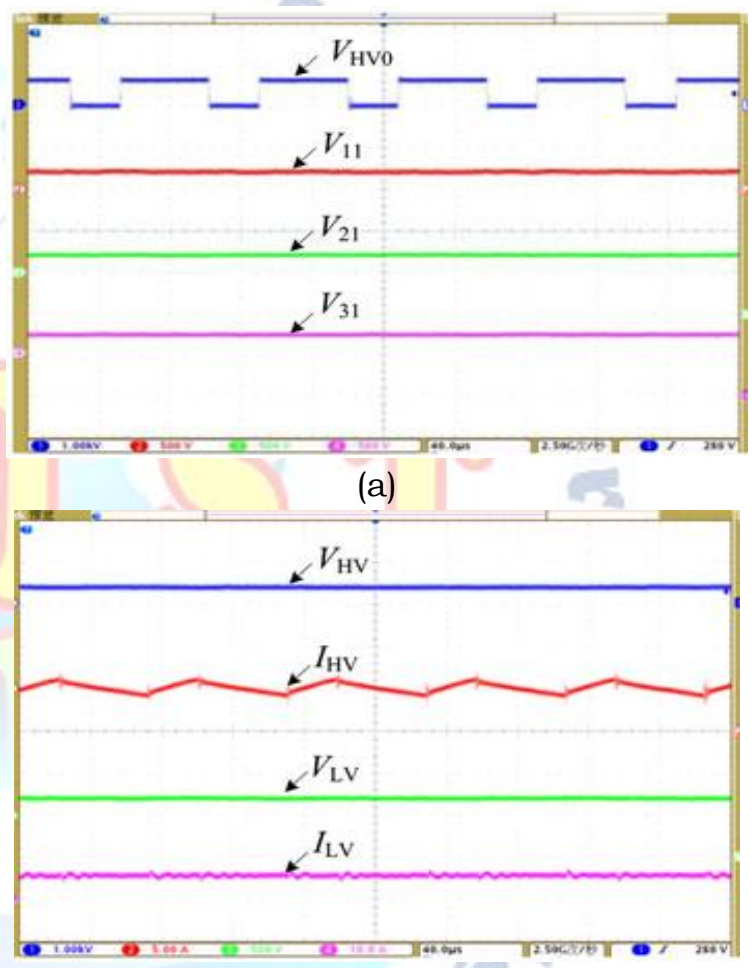

(b)

Fig.7. Voltage and current waveforms of PET (a) $V_{H V 0}, V_{11}, V_{21}$, and $V_{31}$. (b) $V_{H V}, I_{H V}, V_{L V}$, and $I_{L V}$.

From the simulation results, the current stress of PETT remains constant in different conditions and that the current stress of traditional DC transformer increases quickly when the voltage deviate away from the matching value. Due to the addition of series SC modules, the switched capacitor voltages are balanced, but the efficiency of traditional DC transformer may decreases quickly when the voltage deviates away from the matching value, and it is lower than that of PET over a wide range. From Fig.7 simulation results, the PET can adjust the voltage of the dc discrete terminals to ensure the voltage match between the 
two sides of the HFL transformer, and then the current stress and efficiency performances can be improved.

\section{CONCLUSION AND THE FUTURE SCOPE}

The HF DC transformer will be the key device for the future dc power distribution to achieve flexible control and fast management of voltage and power between the MVDC distribution grid and the LVDC microgrid. The PET scheme is proposed in this paper, and the voltage and power characterization, control strategy, and Matlab simulation of PET for MVDC distribution application are present and analyzed in detail. Compared to the traditional DC transformer method, the proposed PET can disconnect from the MVDC distribution grid effectively as a dc breaker and adjust the voltage of the dc discrete terminals to ensure the voltage matches the two sides of the HFL transformer. The circulating current is also decreased and the current and efficiency performances are improved.

Apart from the above, PET can operate well even though some sub modules fail, and also the reliability is improved. The simulation results verify the better performances of the proposed PET, which can satisfy the requirements of practical applications in dc distribution to connect various renewable sources/loads like PV, automotive batteries, wind farms in to the LVDC microgrids.

\section{ACKNOWLEDGMENT}

I would like to express my deep gratitude to my co guide Dr. K. Chiranjeevi, Associate professor, department of Electrical Engineering for his valuable and constructive suggestions towards the completion of this research paper. I would like to acknowledge Battula Greeshma, Scientist-C, ISTRAC, ISRO, for her constructive timely suggestions valuable technical inputs. Not but not least, I am indebted to Prof. B. Subba Reddy towards his valuable contributions in completing this paper.

\section{REFERENCES}

[1] N. Denniston, A. M. Massoud, S. Ahmed, and P. N. Enjeti, "Multiple module high-gain high-voltage dc-dc transformers for offshore wind energy systems," IEEE Trans. Ind. Electron., vol. 58, no. 5, pp. 1877-1886, May 2011.

[2] B. Zhao, Q. Song, J. Li, W. Liu, G. Liu, and Y. Zhao, "High-frequency link dc transformer based on switched capacitor for medium-voltage dc power distribution application," IEEE Trans. Power Electron., vol. 31, no. 7, pp. 4766-4777, Jul. 2016.

[3] Y. Xie, J. Sun, and J. S. Freudenberg, "Power flow characterization of a bidirectional galvanically isolated high-power dc-dc converter over a wide operating range,"
IEEE Trans. Power Electron., vol. 25, no. 1, pp. 54-66, Jan. 2010.

[4] B. Zhao, Q. Song, W. Liu, and Y. Sun, "Overview of dual-active-bridge isolated bidirectional dc-dc converter for high-frequency-link power- conversion system," IEEE Trans. Power Electron., vol. 29, no. 8, pp. 4091-4106, Aug. 2014.

[5] 5. L. He, "High-performance bridge modular switched-capacitor converter with small component requirement based on output impedance analysis for low loss," IEEE Trans. Power Electron., vol. 28, no. 10, pp. 4668-4680, Oct. 2013.

[6] A. Mohammadpour, L. Parsa, M. H. Todorovic, R. Lai, R. Datta, and L. Garces, "Series-input parallel-output modular-phase dc-dc converter with soft switching and high frequency isolation," IEEE Trans. Power Electron., vol. 31, no. 1, pp. 111-119, Jan. 2016.

[7] M. J. Carrizosa, A. Benchaib, P. Alou, and G. Damm, "DC transformer for dc/dc connection in HVDC network," in Proc. 15th European Power Electron. Appl., 2013, pp. 1-10.

[8] L. Hong, Q. Xu, A. Luo, Z. He, Y. Chen and Y. Yue, "Multi-layer fault-tolerant control of MMC for shipboard MVDC power system," 2018 13th IEEE Conference on Industrial Electronics and Applications (ICIEA), Wuhan, China, 2018, pp. 2069-2074.

[9] S. Zhao, Y. Chen, S. Cui, B. J. Mortimer and R. W. De Doncker, "Three-Port Bidirectional Operation Scheme of Modular-Multilevel DC-DC Converters Interconnecting MVDC and LVDC Grids," in IEEE Transactions on Power Electronics, vol. 36, no. 7, pp. 7342-7348, July 2021.

[10] R. Xie and H. Li, "Fault Performance Comparison Study of a Dual Active Bridge (DAB) Converter and an Isolated Modular Multilevel DC/DC (iM2DC) Converter for Power Conversion Module Application in a Breaker-Less Shipboard MVDC System," in IEEE Transactions on Industry Applications, vol. 54, no. 5, pp. 5444-5455, Sept.-Oct. 2018.

[11] L. Lin, G. Cai, C. Liu, Z. Pei, X. Song and J. Wang, "Comparison of MMC Based PET and I-M2C Based PET for Topology, Control Strategy and Cost," 2020 IEEE 9th International Power Electronics and Motion Control Conference (IPEMC2020-ECCE Asia), Nanjing, China, 2020, pp. $2480-2484$

[12] Y. Zhu, S. Shi, S. Cheng, R. Ding, X. Du and F. Zhuo, "Topology, Modulation and Control Strategy of a Multi-port DC/DC Converter based on Modular Multilevel Converter," 2019 IEEE 10th International Symposium on Power Electronics for Distributed Generation Systems (PEDG), Xi'an, China, 2019.

[13] J. Yang et al., "A Multiport Power Electronic Transformer Based on MMC with Resonant Circuit for DC Distribution," 2020 IEEE 9th International Power Electronics and Motion Control Conference (IPEMC2020-ECCE Asia), Nanjing, China, 2020, pp. 945-949.

[14] D. Kong, C. Liu, H. Ying, Z. Pei, J. Li and H. Zhang, "Operating Modes Analysis and Control Strategy of Single-Stage Isolated Modular Multilevel Converter (I-MMC) for Medium Voltage AC/DC Grid," IECON 2019 - 45th Annual Conference of the IEEE Industrial Electronics Society, Lisbon, Portugal, 2019, pp. 5674-5679.

[15] S. Kenzelmann, A. Rufer, D. Dujic, F. Canales, and Y. R. de Novaes, "Isolated $\mathrm{dc} / \mathrm{dc}$ structure based on modular multilevel converter," IEEE Trans. Power Electron., vol. 30, no. 1, pp. 89-98, Jan. 2015. 